\title{
ORTHOGONAL MATCHED FILTER DETECTION
}

\author{
Yonina C. Eldar and Alan V. Oppenheim \\ Research Laboratory of Electronics \\ MIT, Cambridge, MA 02139, USA
}

\begin{abstract}
In this paper we consider the generic problem of detecting a transmitted signal when one of $M$ known signals is transmitted: Instead of using a classical matched filter (MF) detector, matched to the transmitted signals, we propose using an orthogonal matched filter (OMF) detector, which is matched to a set of orthogonal signals that are closest in a least-squares sense to the transmitted signals. We show that this approach is equivalent to optimally whitening the output of the MF demodulator, and then basing the detection on the whitened output. We provide simulation results that suggest that in many cases the OMF detector outperforms the MF detector.
\end{abstract}

\section{INTRODUCTION}

Signal detection in Gaussian and non-Gaussian noise has been studied extensively in the literature (see e.g., [3] and references therein). A generic problem is one of detecting the transmitted signal when one of $M$ known signals is transmitted. The detection is based on the received signal which is typically modeled as the output of an additive noise channel with the transmitted signal as its input.

When the additive noise is white and Gaussian, it is well known (see e.g., [4]) that the optimal signal demodulator consists of a bank of matched filters, referred to as a matched filter (MF) demodulator, followed by an optimal detector that is designed to minimize the probability of error. The detector chooses as the detected signal the one for which the output of the matched filter is maximized. We refer to this combined demodulator and detector as the MF detector.

If the noise is not Gaussian, then the MF detector does not necessarily minimize the probability of error. However, it is still used as the detector of choice in many applications. One justification for its use is that if a signal is corrupted by additive white noise, then the filter matched to that signal maximizes the output signal-to-noise ratio (SNR).

In this paper we propose an orthogonal matched filter (OMF) detector for detecting signals in additive white non-Gaussian noise. Specifically, we propose filtering the signals with a bank of filters matched to a set of orthogonal signals that are closest in a leastsquares sense to the given signals. We show that this approach is equivalent to optimally whitening the output of the MF demodulator, and then basing the detection on the whitened output. We provide simulation results that suggest that in certain cases the OMF detector outperforms the MF detector.

This research was supported in part through collaborative participation in the Advanced Sensors Consortium sponsored by the U.S. Army Research Laboratory under Cooperative Agreement DAAL01-96-2-0001. Yonina Eldar is supported by an IBM Research Fellowship.

\section{PROBLEM FORMULATION}

Suppose we have a transmitter that transmits one of $M$. signals $\left\{s_{m}(t), 1 \leq m \leq M\right\}$ with equal probability, where the signals lie in a real Hilbert space $\mathcal{H}$ with inner product $\langle x(t), y(t)\rangle=$ $\int x(t) y(t) d t$. We assume that the signals are linearly independent and normalized so that $\int s_{m}^{2}(t) d t=1$ for all $m$. The more general case of linearly dependent signals is considered in [1]. The received signal $r(t)$ is modeled as $r(t)=s_{m}(t)+n(t)$, where $n(t)$ is a stationary white noise process with zero mean and spectral density $\sigma^{2}$.

We demodulate the signal $r(t)$ using a correlation demodulator as depicted in Fig. 1: The received signal $r(t)$ is crosscorrelated with $M$ normalized signals $h_{m}(t) \in \mathcal{H}$ so that $a_{m}=$ $\left\langle h_{m}(t), r(t)\right\rangle$, where the signals $h_{m}(t)$ are to be determined. The detected signal is $s_{i}(t)$ where $i=\arg \max a_{m}$. The difference between the OMF detector and the MF detector lies in the choice of the signals $h_{m}(t)$.

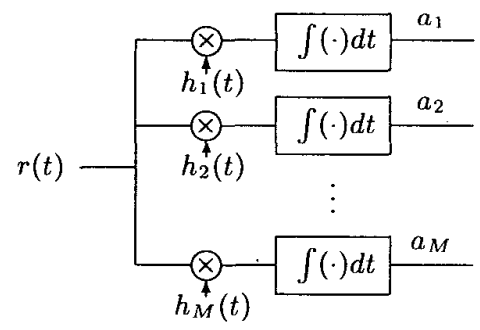

Figure 1: Correlation demodulator.

If the transmitted signal is $s_{i}(t)$, then

$$
a_{m}=\left\langle h_{m}(t), r(t)\right\rangle=\left\langle h_{m}(t), s_{i}(t)\right\rangle+\left\langle h_{m}(t), n(t)\right\rangle .
$$

The detected signal will be the transmitted signal $s_{i}(t)$ if $\max _{m}\left\langle h_{m}(t), s_{i}(t)+n(t)\right\rangle=\left\langle h_{i}(t), s_{i}(t)+n(t)\right\rangle$. Therefore we would like to choose the signals $h_{m}(t)$ to maximize $\left\langle h_{m}(t), s_{m}(t)\right\rangle$ for $1 \leq m \leq M$. It is well known that the signals $h_{m}(t)=s_{m}(t)$ maximize this inner product. The resulting demodulator is then equivalent to the well known MF demodulator [4]. We note that $h_{m}(t)=s_{m}(t)$ also maximizes the sum $R_{h s}=\sum_{m=1}^{M}\left\langle h_{m}(t), s_{m}(t)\right\rangle$, since the individual terms are maximized by this choice. We will see shortly that when additional constraints are imposed it will be useful to consider maximizing the sum rather than the individual terms.

In general, the outputs $a_{m}$ of the demodulator are correlated since they share information regarding the noise process $n(t)$. In- 
tuitively, it seems that eliminating this common (linear) information can improve the performance of the detector. We therefore would like to choose the signals $h_{m}(t)$ so that the outputs $a_{m}$ are uncorrelated. We will show that when the noise is non-Gaussian this approach does in fact lead to improved performance over conventional MF detection in many cases. Then,

Let $\operatorname{cov}\left(a_{m}, a_{k}\right)$ denote the cross-covariance of $a_{m}$ and $a_{k}$.

$$
\begin{aligned}
\operatorname{cov}\left(a_{m}, a_{k}\right) & =E\left(\left\langle h_{m}(t), n(t)\right\rangle\left\langle n(t), h_{k}(t)\right\rangle\right) \\
& =\sigma^{2}\left\langle h_{m}(t), h_{k}(t)\right\rangle .
\end{aligned}
$$

From (2) it follows that the outputs of the demodulator are uncorrelated if and only if the signals $h_{m}(t)$ are orthonormal, i.e., if and only if $\left\langle h_{m}(t), h_{k}(t)\right\rangle=\delta_{m k}$ for all $m, k$. We therefore propose to choose the signals $h_{m}(t)$ to be orthonormal.

As before, we would also like to choose the signals $h_{m}(t)$ to maximize $\left\langle h_{m}(t), s_{m}(t)\right\rangle$ for $1 \leq m \leq M$. However, we now have an additional constraint, namely that the signals $h_{m}(t)$ are orthonormal. If the signals $s_{m}(t)$ are not orthonormal, then we cannot maximize the inner products individually subject to this constraint. Instead, we consider maximizing the sum of the inner products. Thus we seek a set of signals $\left\{h_{m}(t), 1 \leq m \leq M\right\}$ such that

$$
R_{h s}=\sum_{m=1}^{M}\left\langle h_{m}(t), s_{m}(t)\right\rangle
$$

is maximized, subject to the constraint

$$
\left\langle h_{m}(t), h_{k}(t)\right\rangle=\delta_{m k}, 1 \leq m, k \leq M .
$$

\section{EQUIVALENT PROBLEMS}

In this section we formulate the design problem of (3) and (4) in two equivalent ways that provide further insight into the problem. Specifically, we show that the following problems are the same:

1. Find a set of orthonormal signals $\left\{h_{m}(t), 1 \leq m \leq M\right\}$ that maximize $R_{h s}=\sum_{m}\left\langle s_{m}(t), h_{m}(t)\right\rangle$;

2. find an optimal whitening transformation $\mathbf{W}$ that minimizes the total mean squared error (MSE) between the whitened output $\mathbf{b}=\mathbf{W} \tilde{\mathbf{a}}$ and the input $\tilde{\mathbf{a}}$, where ã denotes the vector output of the conventional MF demodulator. Then choose the signals $\left\{h_{m}(t), 1 \leq m \leq M\right\}$ to be the orthonormal signals given by $h_{m}(t)=\sum_{k} \mathbf{W}_{m k}^{*} s_{k}(t)$;

3. find a set of orthonormal signals $\left\{h_{m}(t), 1 \leq m \leq M\right\}$ that are closest in a least-squares sense to the signals $\left\{s_{m}(t), 1 \leq m \leq M\right\}$, namely that minimize $\varepsilon_{l s}=$ $\sum_{m}\left\langle s_{m}(t)-h_{m}(t), s_{m}(t)-h_{m}(t)\right\rangle$.

In section 4 we determine the signals $h_{m}(t)$ through problem 2 above, i.e., by first determining the optimal whitening transformation. Problem 3 has been solved in [2] in the context of quantum detection.

In the remainder of this section we show the equivalence between the three problems above

Let $S: \mathcal{C}^{M} \rightarrow \mathcal{H}$ denote the linear transformation defined by $S \mathbf{x}=\sum_{m=1}^{M} x_{m} s_{m}(t)$, where $\mathbf{x} \in \mathcal{C}^{M}$ is an arbitrary $M$ dimensional vector and $x_{m}$ denotes the $m$ th component of $\mathbf{x}$. Let $S^{*}: \mathcal{H} \rightarrow \mathcal{C}^{M}$ denote the adjoint transformation so that if $\mathbf{x}=$ $S^{*} y(t)$ for arbitrary $y(t) \in \mathcal{H}$, then $x_{m}=\left\langle s_{m}(t), y(t)\right\rangle$. Let $\mathbf{a}$ denote the vector with $m$ th component $\tilde{a}_{m}$, where $\tilde{a}_{m}$ is the output of the correlation demodulator when $h_{m}(t)=s_{m}(t)$. From (2) it follows that the covariance matrix of $\overline{\mathbf{a}}$, denoted $\mathbf{C}_{a}$, is given by

$$
\mathbf{C}_{a}=\sigma^{2} S^{*} S
$$

where the $m k$ th element of $S^{*} S$ is $\left\langle s_{m}(t), s_{k}(t)\right\rangle$. If the signais $s_{m}(t)$ are not orthonormal, then $\mathrm{C}_{a}$ is not diagonal and the elements of $\mathbf{a}$ are correlated. Suppose we whiten ã using a whitening transformation $\mathbf{W}$ to obtain the random vector $\mathbf{b}=\mathbf{W} \tilde{\mathbf{a}}$, where the covariance matrix of $\mathbf{b}$ is given by $\mathbf{C}_{b}=\sigma^{2} \mathbf{I}$, and then base our detection on $\mathbf{b}$. Thus the components $b_{m}$ of $\mathbf{b}$ are the inputs to the detector, and the detected signal is $s_{i}(t)$ if $i=\arg \max b_{m}$. Since the detector bases its decision on the vector $\mathbf{b}$, we choose $\mathbf{a}$ whitening transformation $\mathbf{W}$ that minimizes the MSE given by

$$
\varepsilon_{m s e}=\sum_{m=1}^{M} E\left(\left(b_{m}^{\prime}-\tilde{a}_{m}^{\prime}\right)^{2}\right),
$$

where $\tilde{a}_{m}^{\prime}=\tilde{a}_{m}-E\left(\tilde{a}_{m}\right)$ and $b_{m}^{\prime}=b_{m}-E\left(b_{m}\right)$. That is, from all possible whitening transformations we seek the one that results in a white vector $\mathbf{b}$ as close as possible to the original vector $\tilde{\mathbf{a}}$.

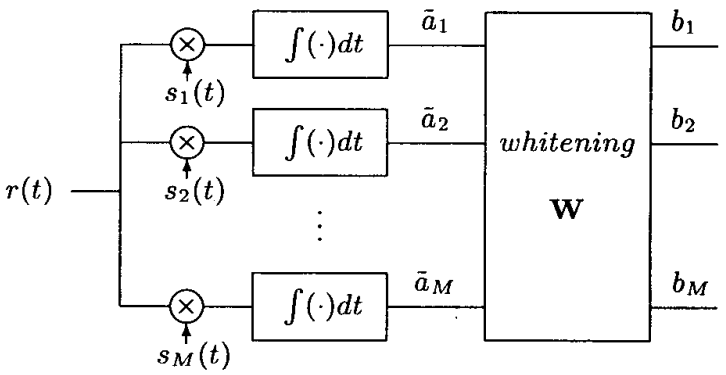

Figure 2: Correlation demodulator followed by whitening.

We now show that the demodulator depicted in Fig. 2 is equivalent to the correlation demodulator of Fig. 1 where the signals $h_{m}(t)$ are orthonormal and given by $h_{m}(t)=\sum_{k} \mathbf{W}_{m k}^{*} s_{k}(t)$, where $\mathbf{W}_{m k}$ denotes the $m k$ th element of $\mathbf{W}$. In other words, the outputs of Fig. 1 and 2 are equal, provided that $h_{m}(t)=$ $\sum_{k} \mathbf{W}_{m k}^{*} s_{k}(t)$.

The output $\mathbf{b}$ of Fig. 2 is given by

$$
\mathbf{b}=\mathbf{W} \tilde{\mathbf{a}}=\mathbf{W} S^{*} r(t)=H^{*} r(t),
$$

where $H: \mathcal{C}^{M} \rightarrow \mathcal{H}$ is given by $H=S \mathbf{W}^{*}$. Therefore, $\mathbf{b}$ can be viewed as the output of a correlation demodulator with signals $h_{m}(t)=\sum_{k} \mathbf{W}_{m k}^{*} s_{k}(t)$.

We now need to show that the signals $h_{m}(t)$ are orthonormal. It is sufficient to show that $H^{*} H=\mathbf{W} S^{*} S \mathbf{W}^{*}=\mathbf{I}$. By definition, $\mathbf{C}_{b}=\sigma^{2} \mathbf{I}$. In addition, $\mathbf{C}_{b}=\mathbf{W} \mathbf{C}_{a} \mathbf{W}^{*}$ and from (5) $\mathbf{C}_{a}=\sigma^{2} S^{*} S$. Therefore $H^{*} H=\mathbf{W} S^{*} S \mathbf{W}^{*}=1 / \sigma^{2} \mathbf{C}_{b}=\mathbf{I}$.

In summary, the output of Fig. 2 may be obtained using the correlation demodulator of Fig. 1, where the signals $h_{m}(t)$ are orthonormal and given by $h_{m}(t)=\sum_{k} \mathbf{W}_{k m}^{*} s_{k}(t)$.

We now show that minimization of $\varepsilon_{m s e}$ given by (6), is equivalent to maximization of $R_{h s}$ given by (3). Using (7) we have

$\mathbf{b}-\tilde{\mathbf{a}}=\left(H^{*}-S^{*}\right) r(t)=\left(H^{*}-S^{*}\right)\left(s_{i}(t)+n(t)\right)$ 
and

$$
b_{m}^{\prime}-\tilde{a}_{m}^{\prime}=\left\langle h_{m}(t)-s_{m}(t), n(t)\right\rangle .
$$

Substituting (9) into (6) we have

$$
\varepsilon_{m s e}=\sum_{m=1}^{M} E\left(\left\langle h_{m}(t)-s_{m}(t), n(t)\right\rangle^{2}\right) .
$$

Let $e_{m}=E\left(\left\langle s_{m}(t)-h_{m}(t), n(t)\right\rangle^{2}\right)$. Then

$$
\begin{aligned}
e_{m} & =E\left(\int_{t}\left(s_{m}(t)-h_{m}(t)\right) n(t) d t\right)^{2} \\
& =\int_{t, t^{\prime}}\left(s_{m}(t)-h_{m}(t)\right)\left(s_{m}\left(t^{\prime}\right)-h_{m}\left(t^{\prime}\right)\right) E\left(n(t) n\left(t^{\prime}\right)\right) d t d t^{\prime} \\
& =\sigma^{2} \int_{t}\left(s_{m}(t)-h_{m}(t)\right)^{2} d t \\
& =\sigma^{2}\left\langle s_{m}(t)-h_{m}(t), s_{m}(t)-h_{m}(t)\right\rangle .
\end{aligned}
$$

Combining (10) and (11) we see that minimization of $\varepsilon_{m s e}$ is equivalent to minimization of $\varepsilon_{l s}$, where

$$
\varepsilon_{l_{s}}=\sum_{m=1}^{M}\left\langle s_{m}(t)-h_{m}(t), s_{m}(t)-h_{m}(t)\right\rangle .
$$

Therefore, the optimal whitening problem is equivalent to the problem of finding a set of orthonormal signals $\left\{h_{m}(t), 1 \leq\right.$ $m \leq M\}$ that are closest in the least-squares sense to the signals $\left\{s_{m}(t), 1 \leq m \leq M\right\}$.

Finally, we show that this least-squares problem is equivalent to our original design problem of (3) and (4). Expanding $\varepsilon_{l s}$ we have

$$
\begin{aligned}
& \varepsilon_{l s}= \\
& =\sum_{m=1}^{M}\left(\left\langle s_{m}(t), s_{m}(t)\right\rangle+\left\langle h_{m}(t), h_{m}(t)\right\rangle-2\left\langle s_{m}(t), h_{m}(t)\right\rangle\right) \\
& =\sum_{m=1}^{M}\left(2-2\left\langle s_{m}(t), h_{m}(t)\right\rangle\right) .
\end{aligned}
$$

From (3) and (13) it follows that minimization of $\varepsilon_{l s}$ is equivalent to maximization of $R_{h s}$. Since minimization of $\varepsilon_{m s e}$ is equivalent to minimization of $\varepsilon_{l s}$, we conclude that these three problems are equivalent.

Note, that if the transmitted signals $s_{m}(t)$ are orthonormal, then the output of the MF demodulator $\overline{\mathbf{a}}$ is white. Thus, in this case $\mathbf{W}=\mathbf{I}$ and the OMF detector is equivalent to the MF detector. Alternatively, if the signals $s_{m}(t)$ are orthonormal then the residual least-squares error $\varepsilon_{l s}$ is minimized when $h_{m}(t)=s_{m}(t)$, and again the OMF detector reduces to the MF detector.

\section{OPTIMAL WHITENING}

Since the optimal whitening problem is equivalent to the problem of (3)-(4), we choose to determine the signals $\left\{h_{m}(t), 1 \leq m \leq M\right\}$ by solving this problem.

We first restate the optimal whitening problem in its most general form. Let a $\in \mathcal{R}^{M}$ be a random vector with $m$ th component $a_{m}$ and positive-definite covariance matrix $\mathbf{C}_{a}$, and let
$a_{m}^{\prime}=a_{m}-E\left(a_{m}\right)$. We seek a whitening transformation $\mathbf{W}$ such that the white vector $\mathbf{b}=$ Wa has a covariance matrix $\mathbf{C}_{b}=\sigma^{2} \mathbf{I}$, and is as close as possible to $a$ in the MSE sense. Thus, we seek the transformation $W$ that minimizes

$$
\varepsilon_{m s e}=\sum_{m=1}^{M} E\left(\left(a_{m}^{\prime}-b_{m}^{\prime}\right)^{2}\right),
$$

where $b_{m}$ is the $m$ th component of $\mathbf{b}$, and $b_{m}^{\prime}=b_{m}-E\left(b_{m}\right)$, subject to the constraint

$$
\mathbf{C}_{b}=\mathbf{W C}_{a} \mathbf{W}^{*}=\sigma^{2} \mathbf{I}
$$

where $\mathbf{C}_{b}$ is the covariance matrix of $\mathbf{b}$. Since $\mathbf{W}$ must be invertible (15) reduces to

$$
\sigma^{2}\left(\mathbf{W}^{*} \mathbf{W}\right)^{-1}=\mathbf{C}_{a} .
$$

We solve this minimization problem using the eigendecomposition of $\mathbf{C}_{a}$ and the singular value decomposition (SVD) [5] of W. so that

Let the vectors $\mathbf{v}_{k}$ denote the orthonormal eigenvectors of $\mathbf{C}_{a}$,

$$
\mathbf{C}_{a} \mathbf{v}_{k}=\lambda_{k} \mathbf{v}_{k}, 1 \leq k \leq M
$$

where $\lambda_{k}>0$. We can then decompose $\mathbf{C}_{a}$ as $\mathbf{C}_{a}=\mathrm{VDV}^{*}$ where $\mathbf{V}$ denotes the unitary matrix of columns $\mathbf{v}_{k}$, and $\mathbf{D}$ denotes the diagonal matrix with diagonal elements $\lambda_{k}$. Then

$$
\mathbf{W}^{*} \mathbf{W} \mathbf{v}_{k}=\sigma^{2} \mathbf{C}_{a}^{-1} \mathbf{v}_{k}=\sigma^{2} \lambda_{k}^{-1} \mathbf{v}_{k} .
$$

From the properties of the SVD (see, e.g. [5]) it then follows that

$$
\mathbf{W} \mathbf{v}_{k}=\sigma_{k} \mathbf{u}_{k}, 1 \leq k \leq M
$$

where $\sigma_{k}=\sigma / \sqrt{\lambda_{k}}$ and the vectors $\mathbf{u}_{k}$ are orthonormal.

Since the $M$ vectors $\mathrm{v}_{k} \in \mathcal{C}^{M}$ are orthonormal, they. span the space $\mathcal{C}^{M}$, and any $\mathbf{x} \in \mathcal{C}^{M}$ may be expressed as $\mathbf{x}=$ $\sum_{k}\left\langle\mathbf{v}_{k}, \mathbf{x}\right\rangle \mathbf{v}_{k}$, where the inner product on $\mathcal{C}^{M}$ is defined as $\left\langle\mathbf{v}_{k}, \mathbf{x}\right\rangle=\mathbf{v}_{k}^{*} \mathbf{x}$. Let $\mathbf{a}^{\prime}=\mathbf{a}-E(\mathbf{a})$ and $\mathbf{b}^{\prime}=\mathbf{b}-E(\mathbf{b})$. Then $\mathbf{a}^{\prime}=\sum_{k}\left\langle\mathbf{v}_{k}, \mathbf{a}^{\prime}\right\rangle \mathbf{v}_{k}$ and

$$
\mathbf{b}^{\prime}-\mathbf{a}^{\prime}=\mathbf{W a}^{\prime}-\mathbf{a}^{\prime}=\sum_{k}\left\langle\mathbf{v}_{k}, \mathbf{a}^{\prime}\right\rangle\left(\sigma_{k} \mathbf{u}_{k}-\mathbf{v}_{k}\right)
$$

where we used (19). We can now express $\varepsilon_{m s e}$ of (14) as

$$
\begin{aligned}
& \varepsilon_{m s e}=E\left(\left\langle\mathbf{b}^{\prime}-\mathbf{a}^{\prime}, \mathbf{b}^{\prime}-\mathbf{a}^{\prime}\right\rangle^{2}\right) \\
& \quad=\sum_{k, m} E\left(\left\langle\mathbf{v}_{m}, \mathbf{a}^{\prime}\right\rangle\left\langle\mathbf{a}^{\prime}, \mathbf{v}_{k}\right\rangle\right)\left\langle\sigma_{k} \mathbf{u}_{k}-\mathbf{v}_{k}, \sigma_{m} \mathbf{u}_{m}-\mathbf{v}_{m}\right\rangle
\end{aligned}
$$

Now,

$$
E\left(\left\langle\mathbf{v}_{m}, \mathbf{a}^{\prime}\right\rangle\left\langle\mathbf{a}^{\prime}, \mathbf{v}_{k}\right\rangle\right)=\left\langle\mathbf{v}_{m}, \mathbf{C}_{a} \mathbf{v}_{k}\right\rangle=\lambda_{k}\left\langle\mathbf{v}_{m}, \mathbf{v}_{k}\right\rangle=\lambda_{k} \delta_{m k} .
$$

Substituting (22) in (21) results in

$$
\begin{aligned}
\varepsilon_{m s e} & =\sum_{k} \lambda_{k}\left\langle\sigma_{k} \mathbf{u}_{k}-\mathbf{v}_{k}, \sigma_{k} \mathbf{u}_{k}-\mathbf{v}_{k}\right\rangle \\
& =\sum_{k} \lambda_{k}\left(\sigma_{k}^{2}+1-2 \sigma_{k} \Re\left(\left\langle\mathbf{u}_{k}, \mathbf{v}_{k}\right\rangle\right)\right),
\end{aligned}
$$

where $\Re(\cdot)$ denotes the real part. From (23) it follows that minimizing $\varepsilon_{m s e}$ is equivalent to maximizing $A=$ 
$\sum_{k} \sigma_{k} \Re\left(\left\langle\mathbf{u}_{k}, \mathbf{v}_{k}\right\rangle\right)$. Using the Cauchy-Schwartz inequality we have,

$$
\begin{aligned}
A & =\sum_{k} \sigma_{k} \Re\left(\left\langle\mathbf{u}_{k}, \mathbf{v}_{k}\right\rangle\right) \\
& \leq \sum_{k} \sigma_{k}\left|\left\langle\mathbf{u}_{k}, \mathbf{v}_{k}\right\rangle\right| \\
& \leq \sum_{k} \sigma_{k}\left(\left\langle\mathbf{u}_{k}, \mathbf{u}_{k}\right\rangle\left\langle\mathbf{v}_{k}, \mathbf{v}_{k}\right\rangle\right)^{1 / 2}=\sum_{k} \sigma_{k}
\end{aligned}
$$

with equality in (25) if and only if $\left\langle\mathbf{u}_{k}, \mathbf{v}_{k}\right\rangle$ is real and nonnegative, and equality in (26) if and only if $\mathbf{u}_{k}=c_{k} \mathbf{v}_{k}$ for some nonzero constants $c_{k}$. Since the vectors $\mathbf{u}_{k}$ are orthonormal $\left\langle\mathbf{u}_{k}, \mathbf{u}_{k}\right\rangle=1$. We therefore conclude that $A \leq \sum_{k} \sigma_{k}$ with equality if and only if $\mathbf{u}_{k}=\mathbf{v}_{k}$. Thus $\varepsilon_{m s e}$ is minimized when $\mathbf{W}$ is given by

$$
\mathbf{W} \mathbf{v}_{k}=\sigma_{k} \mathbf{v}_{k}=\frac{\sigma}{\sqrt{\lambda_{k}}} \mathbf{v}_{k}
$$

or

$$
\mathbf{W}=\sigma \mathbf{V} \mathbf{D}^{-1 / 2} \mathbf{V}^{*}=\sigma \mathbf{C}_{a}{ }^{-1 / 2}
$$

In summary, the optimal whitening transformation that minimizes the MSE $\varepsilon_{m s e}$ defined in (14) for an input a with covariance $\mathbf{C}_{a}$ and an output $\mathbf{b}$ with covariance $\mathbf{C}_{b}=\sigma^{2} \mathbf{I}$, is $\mathbf{W}=\sigma \mathbf{C}_{a}{ }^{-1 / 2}$

In Fig. 2 the input to the whitening transformation is $\mathbf{a}=\tilde{\mathbf{a}}$ with $\mathrm{C}_{a}=\sigma^{2} S^{*} S$. Thus, the optimal whitening transformation in this case is $\mathbf{W}=\left(S^{*} S\right)^{-1 / 2}$, and the optimal orthonormal signals $h_{m}(t)$ that maximize $R_{h s}$ are given by $h_{m}(t)=\sum_{k} \mathbf{W}_{m k}^{*} s_{k}(t)$, or $h_{m}(t)=H i_{m}$ where $H=S\left(S^{*} S\right)^{-1 / 2}$ and $i_{m}(k)=\delta_{m k}$.

\section{SIMULATION RESULTS}

In this section we provide simulation results suggesting the behavior and performance of the OMF detector, in comparison to the MF detector.

We compared the performance of the $\mathrm{OMF}$ and MF detectors for random signal constellations. Specifically, we generated random covariance matrices $\mathbf{C}_{a}=\sigma^{2} S^{*} S$ with uniformly distributed elements. We then generated 1000 realizations of random noise vectors (from a given distribution) with zero mean and covariance $\boldsymbol{C}_{a}$, for each realization of $\mathbf{C}_{a}$. The vector output of the MF demodulator was then taken to be the sum of one of the columns of $\mathbf{C}_{a}$ and the random noise. To obtain the output of the OMF detector, the output of the MF demodulator was whitened using the whitening transformation $\mathbf{W}=\sigma \mathbf{C}_{\boldsymbol{a}}{ }^{-1 / 2}$. We then determined the probability of error for both the MF and the OMF detector by recording the number of successful detections.

In Table 1 we show the fraction of the simulations for which the ratio of the probabilities of error using the MF and OMF detectors was found to be in the given ranges. We denote the probability of error using the OMF and the MF detectors by $P_{o}$ and $P$, respectively. The results are shown for three noise distributions, (a) a Gaussian mixture of two components with variance .2 centered at \pm 1 , (b) a Beta distribution with parameters $A=.1$ and $B=.1$, and (c) a discrete-time signal taking on the values \pm 1 with equal probability. The parameters of the distributions were chosen such that the SNR is $0 \mathrm{db}$. For each distribution we evaluated the probability of error by generated 1000 random covariance matrices $C_{a}$, and corresponding to each $\mathbf{C}_{a}$ we generated 1000 random noise vectors with zero mean and covariance $\mathbf{C}_{a}$. The probability of error

\begin{tabular}{c||c|c|c|} 
& $\begin{array}{c}\text { Gaussian } \\
\text { Mixture }\end{array}$ & Beta & $\begin{array}{c} \pm 1 \text { with } \\
\text { equal prob. }\end{array}$ \\
\hline \hline$P_{o} / P<.65$ & 0 & 0 & 0 \\
$.65<P_{o} / P<.95$ & 0.075 & 0.013 & 0.323 \\
$.85<P_{o} / P<.95$ & 0.835 & 0.755 & 0.532 \\
$.95<P_{o} / P<1.05$ & 0.090 & 0.232 & 0.145 \\
$1.05<P_{o}$ & 0 & 0 & 0
\end{tabular}

Table 1: Comparison between the OMF and the MF detectors. $P_{o}$ and $P$ denote the probability of error using the OMF detector and the MF detector, respectively. For each noise distribution we show the fraction of the simulations that resulted in relative probabilities of error in the given ranges.

for each distribution was determined as the fraction of successful detections. The simulation results summarized in Table 1 suggest that the OMF detector outperforms the MF detector in many cases. The simulations were repeated for various SNRs and the results indicate that the relative improvement in performance of the OMF detector over the MF detector increases with increasing SNR.

More extensive simulations are presented in [1], which strongly suggest that when the additive noise is non-Gaussian the OMF detector can significantly decrease the probability of error over the MF receiver and when the additive noise is Gaussian, the degradation in performance using the OMF detector is minor.

\section{CONCLUSION}

We considered the problem of detecting a transmitted signal when one of $M$ known signals is transmitted. We proposed an orthogonal matched filter detector, which can be viewed as a MF demodulator followed by optimal whitening of the demodulators output. Alternatively, we may view the OMF detector as a bank of filters matched to a set of orthogonal signals that are closest in the leastsquares sense to the transmitted signals. We provided simulations that suggest that the OMF detector outperforms the MF detector in many cases.

\section{ACKNOWLEDGMENT}

The authors would like to thank Mr. Petros Boufounos for his help with developing and carrying out the simulations reported here.

\section{REFERENCES}

[1] Y. C. Eldar, A. V. Oppenheim and Dianne Egnor, "Orthogonal and projected orthogonal matched filter detection," submitted to IEEE Trans. on Signal Proc., Jan. 2001.

[2] Y. C. Eldar and G. D. Forney, Jr., "On quantum detection and the square-root measurement," IEEE Trans. on Inform. Theory, Mar. 2001.

[3] T. Kailath and V. Poor, "Detection of stochastic processes," IEEE Trans. on Inform. Theory, vol. 44, pp. 2230-2259, Oct. 1998.

[4] J. G. Proakis, Digital Communications. McGraw-Hill, Inc., third edition, 1995.

[5] G. H. Golub and C. F. Van Loan, Matrix Computations. Johns Hopkins University Press, 1983. 